\title{
How to build patient-specific synthetic abdominal anatomies. An innovative approach from physical toward hybrid surgical simulators
}

\author{
S. Condino ${ }^{1}$ \\ M. Carbone ${ }^{1,4 *}$ \\ V. Ferrari ${ }^{1}$ \\ L. Faggioni ${ }^{2}$ \\ A. Peri $^{3}$ \\ M. Ferrari ${ }^{1}$ \\ F. Mosca ${ }^{1}$ \\ ${ }^{1}$ EndoCAS Center, Università di Pisa, \\ Italy \\ ${ }^{2}$ Divisione di Radiologia Diagnostica e \\ Interventistica, Università di Pisa, \\ Italy \\ ${ }^{3}$ Fondazione IRCCS Policlinico San \\ Matteo Pavia, Università di Pavia, \\ Italy \\ ${ }^{4}$ Scuola Superiore Sant'Anna, Pisa, \\ Italy \\ *Correspondence to: M. Carbone, \\ EndoCAS Center, Università di Pisa, \\ Ospedale di Cisanello, Via Paradisa \\ 2, 56124 Pisa, Italy. \\ E-mail: \\ marina.carbone@endocas.org
}

\begin{abstract}
Background According to literature evidence, simulation is of the utmost importance for training and innovative surgical strategies assessment. At present commercial physical simulators are limited to single or only a few anatomical structures and these are often just standard anatomies.
\end{abstract}

Methods This paper describes a strategy to produce patient-specific abdominal silicone organs with realistic shapes and colors, starting from radiological images. Synthetic organs can be assembled in a complex physical simulator or, if paired with electromagnetic sensors, in a hybrid environment (mixed reality) to quantify deformations caused by surgical action.

Results A physical trunk phantom with liver, gallbladder, pancreas and a sensorized stomach has been developed. It is coupled with consistent radiological images and a 3D model of the entire upper abdomen. The simulator has been evaluated in quantitative and qualitative terms to quantify its accuracy and utility, respectively.

Conclusions This simulator can be used in the field of abdominal surgery to train students and as a testing environment to assess and validate innovative surgical technologies. Copyright $\odot 2011$ John Wiley \& Sons, Ltd.

Keywords patient-specific simulator; physical simulators; hybrid simulators; segmentation; surgical training; abdominal surgery; mixed reality

\section{Introduction}

The development of simulation technologies in the surgical field has been strongly promoted by the advent of minimally invasive surgery which requires particular psychomotor skills and high levels of hand-eye coordination.

In the last decade many types of simulators of varying complexity have been developed and marketed. The existing trainers can be divided into three groups: virtual reality (VR), physical and hybrid simulators $(1,2)$.

Virtual reality simulators combine a convincing virtual representation of an entire organ or a complete body region and an interface to interact with it. An important feature of VR simulators is that they can provide objective and repeated measurements to evaluate the trainees' expensive but they are theoretically optimal to perform destructive tasks. They simulate a virtual scenario that can be reset at the beginning of each trial without needing to buy new phantoms or change spare parts. Furthermore, VR allows easy 
change of the anatomy, offering young surgeons the possibility to try the intervention not strictly on a single standard anatomy. Although during the last decade many companies have proposed virtual simulators, there are still well-described technical challenges to be overcome to permit varied training in a realistic computer generated environment. These challenges include the development of realistic surgical interfaces and environments, the modelling of realistic interactions between objects, and the rendering of the surgical field (4). Moreover, the state of the art for interactive deformable object simulation is still a controversial issue regarding haptic feedback because of the difficulty of incorporating it in a reliable and robust way (5).

Today, excellent results are obtained in the VR simulation of endoscopies (6-8) or endovascular treatments $(9,10)$, where the anatomies are simple tubular structures and there are no complex tasks to simulate.

Simulation using physical objects usually involves plastic, rubber, and latex models arranged in boxes. These objects are used to render different organs and pathologies and they allow one to perform specific tasks such as cutting, suturing, grasping or clipping. Physical simulators are nowadays often restricted to one or a few anatomical structures and they are frequently limited to a standard anatomy. Another limit is related to destructive tasks that require a new, and usually expensive, phantom for each trial. On the other hand, the actual interaction with the simulated anatomy can be considered the principal advantage of physical simulators (11-13).

Conversely, hybrid simulators combine synthetic models with VR by deploying mixed-reality, often by using a realistic interface (such as real diagnostic or surgical instruments) to bridge the gap between the synthetic mannequin and the computer. This avoids some of the technical difficulties associated with reproducing the feel of the instruments and of the human tissue, while still allowing access to the advantages of computer simulation, in particular for trainee performance evaluation, the possibility to enrich the scene with virtual elements and to give instructions for surgical tasks execution (14). These kinds of simulators require sensors to acquire pose and configuration of real structures.

Several papers in the literature aim to identify the level of simulation complexity and realism necessary for efficient training or in vitro evaluation of new technologies (15). Nowadays the increasing realism of visual rendering on one hand, and the availability of soft tissue-like materials on the other, has enabled commercial simulators to acquire some maturity. Although they are attractive, these simulators are limited to the simulation of restricted and determined models within a limited database, and they still lack the realism that can only be reached with patient-specific simulation. At present, in fact, only one company (SimbionixTM in partnership with PolyDimensions $\mathrm{GmbH}$ ) proposes a patient-specific simulation for endoscopic arterial pathologies (16). A few reported works also propose a preoperative patient-specific virtual simulation (17), essentially of laparoscopic procedures (18), orthopaedic surgery (19-22), or vascular interventions $(23,24)$; of these only one (24) proposes a physical simulator.

This paper describes a strategy to build patient-specific physical simulators with basic functions for future hybrid simulation. The idea is to overcome the limits imposed by standard anatomies, starting from the elaboration of radiological images to develop a simulator which includes realistic synthetic organs paired with electromagnetic position sensors and enriched with consistent virtual models of the entire abdomen.

\section{Materials and Methods}

The development of phantom synthetic parts begins with the segmentation and surface extraction of anatomical components from real medical image data sets. Subsequently, rapid prototyping (Dimension Elite 3D Printer (25)) is used for the manufacture of moulds that are employed to obtain soft material (silicone) replica organs. A commercial torso phantom (CLA ${ }^{\circledR}$ OGI Phantom) is used to enfold those models in a realistic environment (14). Supporting structures are designed to guarantee the correct positioning of the synthetic models inside the mannequin and they replicate space constraint and relationships between organs.

The procedure developed enables one to insert sensors inside the organ models. In this work, NDI Aurora ${ }^{\circledR}$ electromagnetic (EM) tracking sensors were used (Aurora ${ }^{\circledR}$ 5DOF Sensor, $0.5 \mathrm{~mm} \times 8 \mathrm{~mm}, 2 \mathrm{~m}$ ) (26). By knowing the precise position of these sensors on the organ models, it is possible to develop a virtual environment that shows possible deformations impressed on organs during surgical tasks in real time.

The following paragraphs detail the three principal production phases:

- images acquisition and elaboration for 3D virtual models extraction;

- fabrication of synthetic organs (with possible inclusion of sensors);

- assembly of replica organs inside the mannequin.

The evaluation steps are described at the end of the paragraph.

\section{Image acquisition and elaboration}

Volumetric radiological images offer high quality anatomy description that can be used not only for diagnosis purposes but also to create realistic patient-specific 3D models. To do this it is necessary to obtain high quality images in terms of contrast and spatial resolution and then to extract the geometrical descriptions of the organs through segmentation. Thus, 25 anonymized MultiDetector Computed Tomography (MDCT) data sets, acquired for standard radiological examination in our 
hospital, were analyzed and segmented. Between these one was selected as optimal to build the simulator.

The selected MDCT was acquired with the following specifications:

- administration of effervescent powder $\left(\mathrm{NaHCO}_{3} / \mathrm{H}_{3} \mathrm{C}_{6}\right.$ $\mathrm{H}_{5} \mathrm{O}_{7}$ ) to inflate the stomach (27);

- at least a basal +2 post-contrastographic acquisition (arterial and venous) to extract each structure from its most contrasted phase;

- slice thickness $\leq 1.2 \mathrm{~mm}$ to obtain optimal resolution of tiny structures.

These parameters can be considered the optimal protocol to obtain virtual starting models. It is very important to consider that, except for administration of the gas contrast, which has no side effects on the patient (it only dilates the stomach), no particularly tailored examinations are needed.

Segmentation was applied to the chosen data set to obtain organ frontiers. For this purpose, a semiautomatic tool, the EndoCAS Segmentation Pipeline (28) integrated in the open source software ITK-SNAP 1.5 (www.itksnap.org) (29) was used.

The whole segmentation procedure is based on the neighbourhood connected region growing algorithm that, appropriately parameterized for the specific anatomy and combined with the optimal segmentation sequence proposed, allows optimal segmentation results without losing simplicity and usability. This basic algorithm is efficient for clinical purposes, in terms of usability, segmentation time, and quality of the results (Figure 1).

The pipeline has been developed for abdominal surgical planning purposes (28), and in this work it has been tailored to the extraction of liver and stomach too.

Figure 2 shows the segmentation process for the stomach.

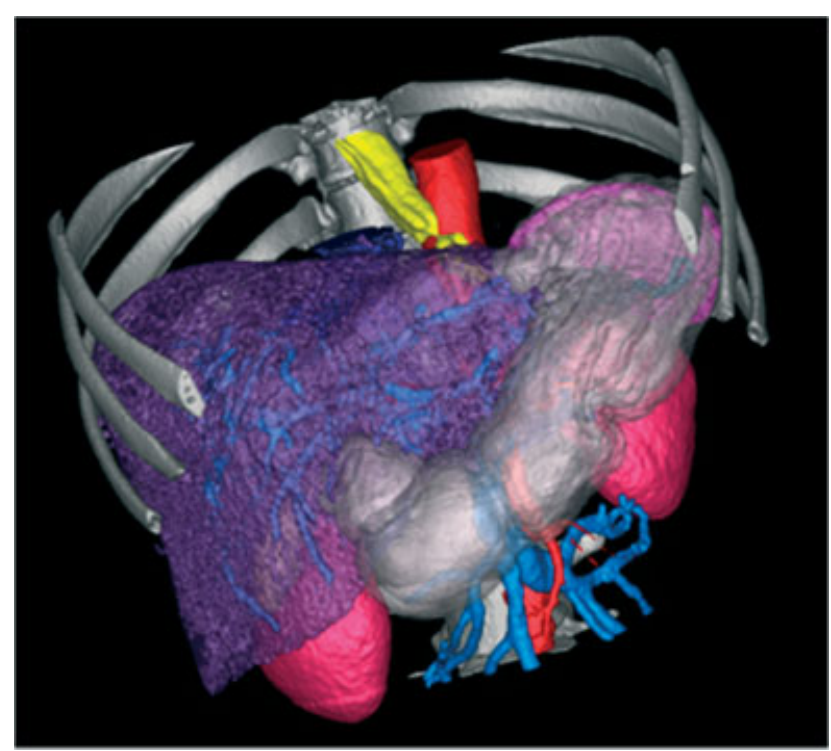

Figure 1. 3D upper abdomen model

\section{Fabrication of synthetic organs}

Silicone rubbers and an agarose gel were employed to fabricate the synthetic organs. Three types of silicone were used: pourable, mouldable, and paintable silicones.

As pourable silicone GLS 50 (Prochima ${ }^{\circledR}(30)$ ), a liquid rubber for casting with a cold cure by poly-condensation, was used; this kind of silicone is suitable for detailed objects and is ideal for the manufacture of small objects.

GSP 400 (Prochima ${ }^{\circledR}$ ) was employed as mouldable silicone; it is a silicone rubber for large sized objects that can be directly applied by hand. Finally, RTV TIXO was used as paintable silicone. This silicone is smooth and thixotropic, and it allows fine reproduction of details. The advantage of the latter two silicones is that they can be applied quickly and they do not require complex moulds.

Pigment powders from Prochima ${ }^{\circledR}$ were mixed with silicones to reproduce realistic color.

To simulate organ consistency (i.e. liver) it was decided to use not only silicone rubbers but also an agarose gel since its mechanical properties closely mimic that of soft tissue (31).

The following paragraphs describe four procedures to reproduce different anatomical structures, possibly sensorized.

The organs in parentheses are selected as examples to explain the various fabrication procedures:

1. hollow organs (gallbladder);

2. sensorized hollow organs (stomach);

3. solid organs (pancreas);

4. sensorized solid organs (liver).

\section{Hollow organs model}

A strategy for hollow organ reproduction is to design a mould consisting of two outer shells and an inner core for pourable silicone injection. This strategy has been used for the gallbladder. The segmented gallbladder and the related 3D model is shown in Figure 3 (left), while Figure 3 (right) shows the mould design.

The mould was fabricated using the $3 \mathrm{D}$ printer, Figure 4(a). The silicone (GLS50) was poured into the assembled mould Figure 4(b). After complete silicone curing, the elastic model of the gallbladder was extracted (unfolded) from the mould Figure 4(c).

An analogous strategy could be used to obtain a gastric model with Elastosil M $4400^{\mathrm{TM}}(32)$, a pourable silicone that has a distensibility similar to that of the human stomach (silicone elongation at break 250\%, human stomach elongation at break about 225\%) and has already been used in earlier studies for the reproduction of a gastric model fabricated starting from a real CT data set (33).

\section{Sensorized hollow organs}

This process allows sensors to be embedded inside the organ wall between two layers of silicone. The following 


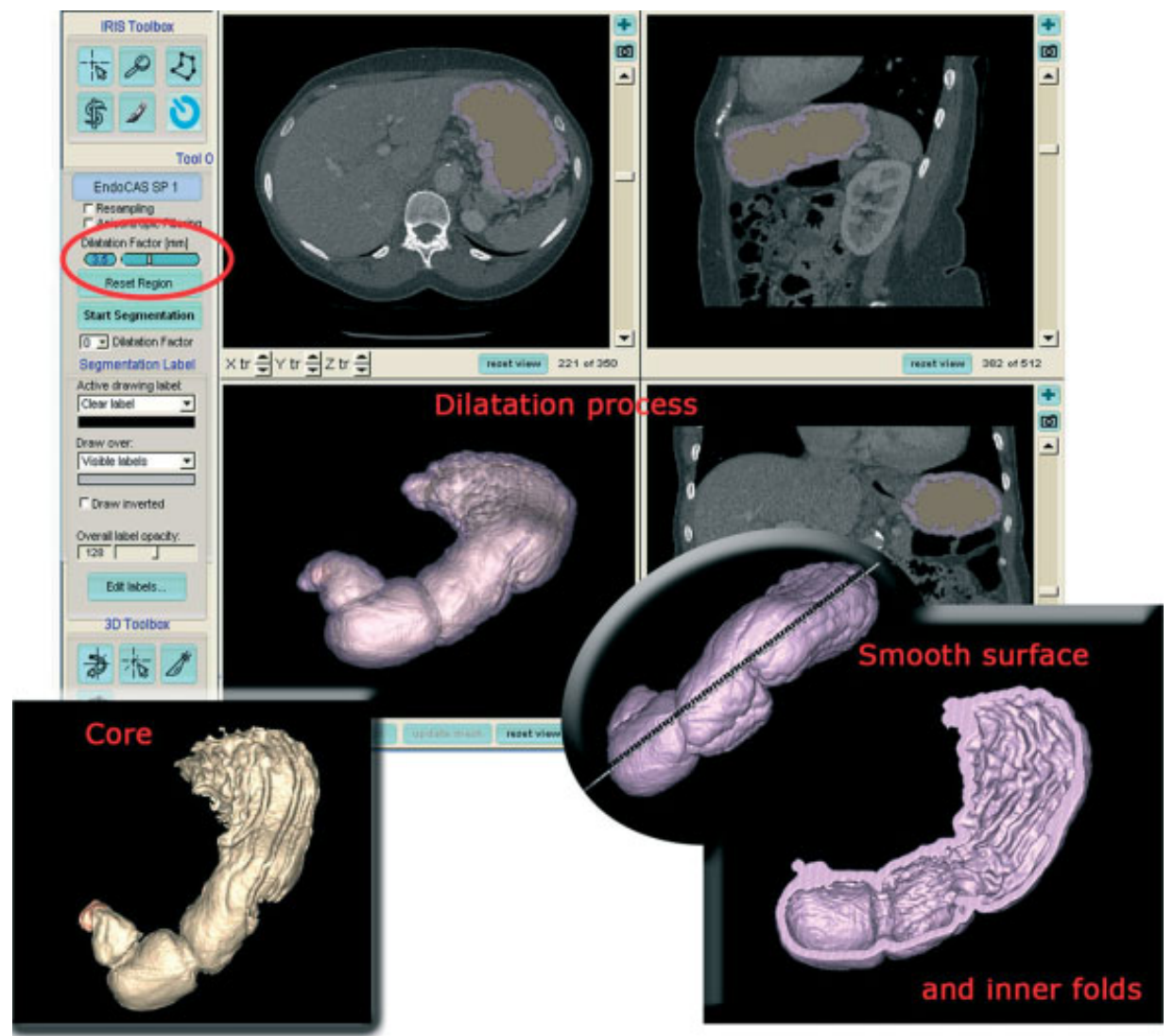

Figure 2. Segmentation process for the stomach: the first step is to segment the inside of the organ (very quickly thanks to the high rate of contrast obtained through the gas). The second step is to segment the organ wall: the software allows one to isotropically dilate the previously segmented area, a final $3.5 \mathrm{~mm}$ dilatation was reached to obtain a smooth surface while maintaining inner gastric folds

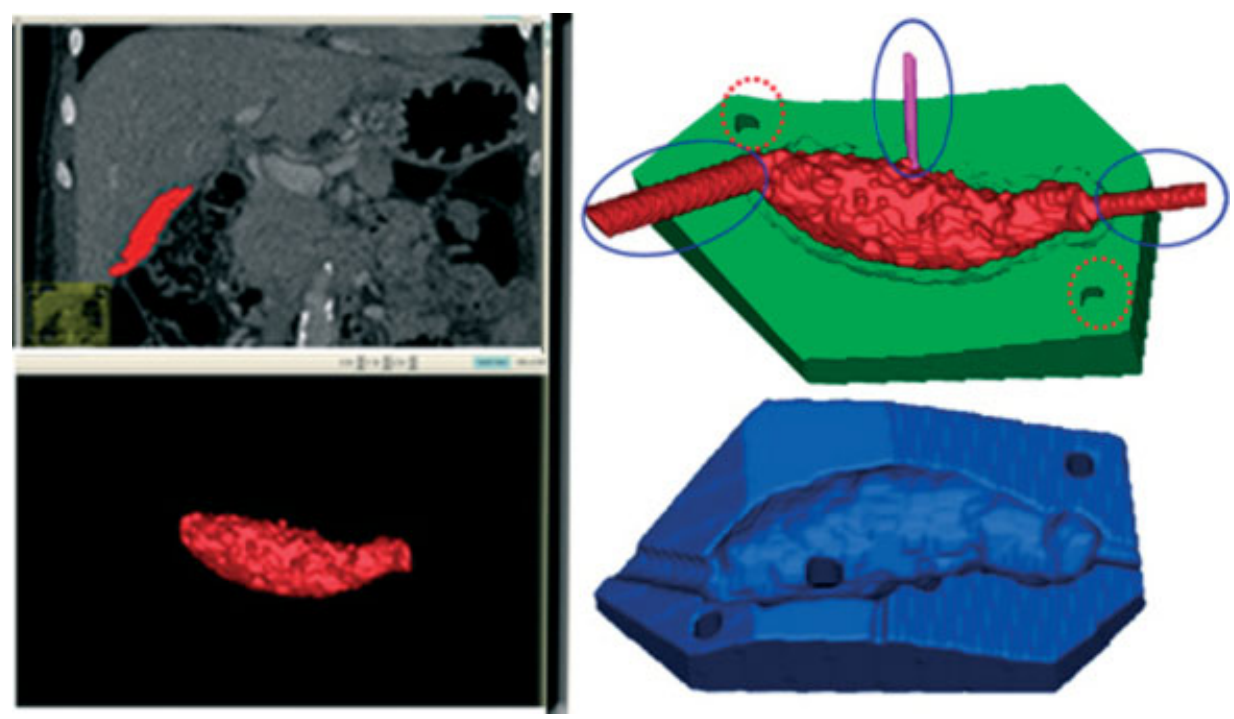

Figure 3. Left - segmented gallbladder and 3D surface rendering. Right - designed gallbladder mould: two external valves and core; the mould inner core coincides with the organ lumen, with two supporting structures and a hole to insert a screw (magenta cylinder) to maintain it in position inside the assembled mould. Similar holes (indicated with dotted red lines) have been predisposed in the mould external parts for their proper alignment

details the procedure for fabricating a sensorized gastric model.

First, the positions of eight Aurora electromagnetic sensors were identified on the 3D virtual model based on the clinicians' needs. Then a mould replicating the gastric lumen was made, with holes corresponding with the planned sensor positions. Figure 5 shows the gastric mould with planned, in virtual Figure 5(a), and actual 


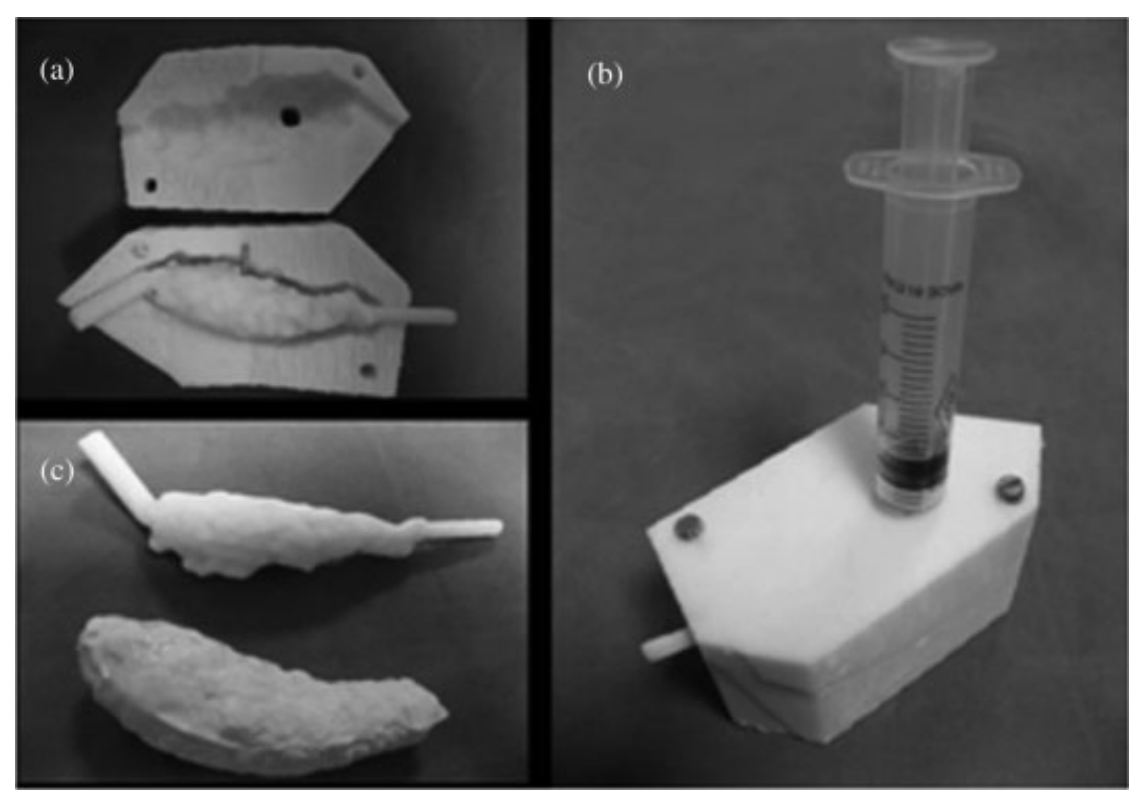

Figure 4. (A) Moulds parts fabricated with the 3D printer. (B) Assembled mould ready for injection of the silicone with a syringe. (C) The finished silicone model near the mould core

screws positioning used for exact sensors positioning Figure 5(b). Figure 5(c) shows a first layer of silicone RTV TIXO applied to the gastric model. After silicone curing, Aurora sensors were positioned between each pair of screws (5(d)); the thin screws were removed from the rigid gastric model and a final layer of GSP 400 (5(e)) was applied.

RTV TIXO was chosen to reproduce the fine gastric folds, and the outer layer of the model was fabricated

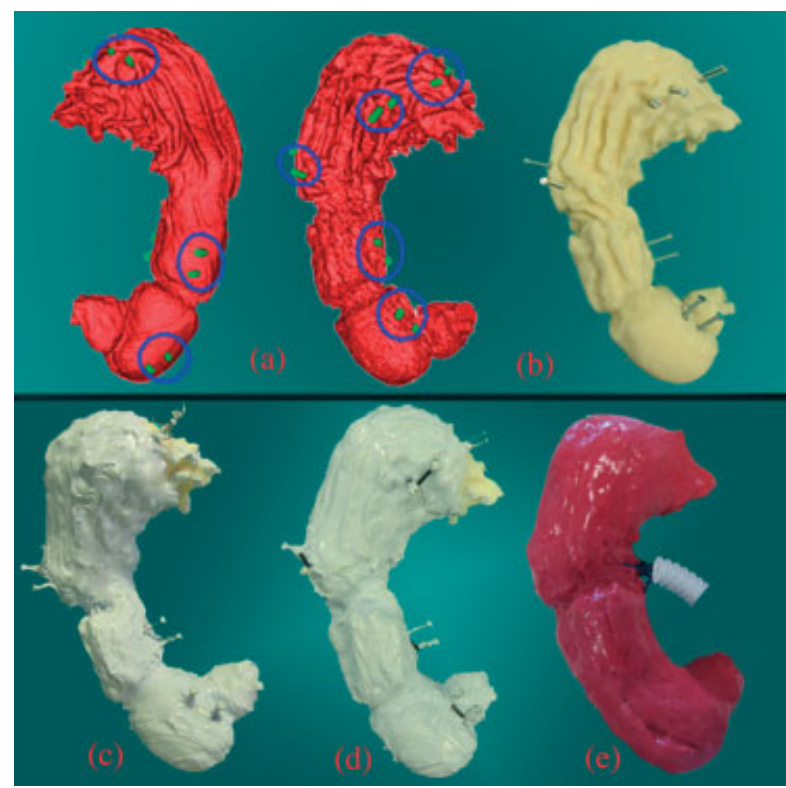

Figure 5. (a) positions for eight Aurora sensors. The green cylinders define the exact position and orientation of each sensor (each pair of cylinders defines the position of the axis of one sensor). (b) A hole for placement of a screw predisposed in the virtual model corresponding with each cylinder. (c) RTV TIXO layer. (d) Aurora sensors positioned between each pair of screws. (e) Final layer of GSP 400

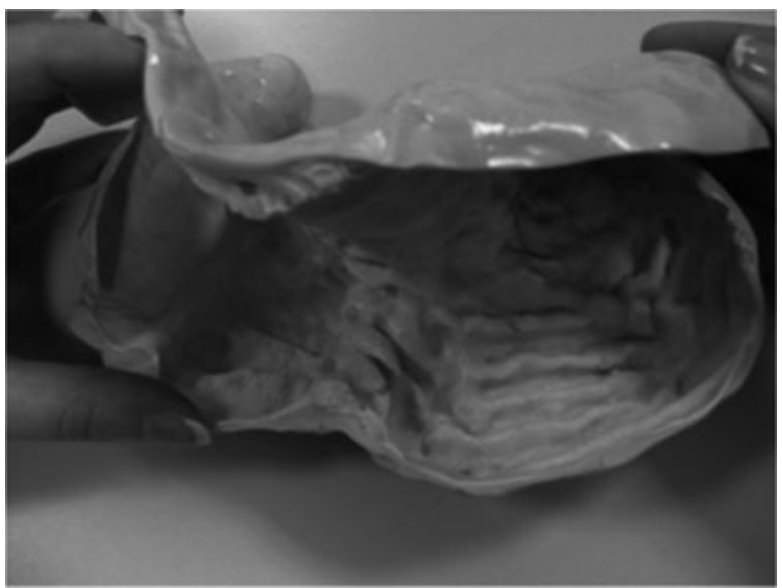

Figure 6. Internal surface of a cut stomach model

using GSP 400, which allows a more uniform and smooth surface. Figure 6 shows the inside of a silicone model that realistically mimics the internal gastric surface.

\section{Solid organs}

A strategy for the reproduction of solid organs is to design a mould composed of an external shell (consisting of two or more joinable parts) that is a negative copy of the organ. This strategy has been used for pancreas reproduction. The designed mould and the resulting silicone pancreas model are shown in Figure 7.

\section{Sensorized solid organ}

This process is similar to the previous one and allows sensors to be embedded inside a silicone shell. This shell can be filled with more appropriate material to reproduce specific organ consistency. 


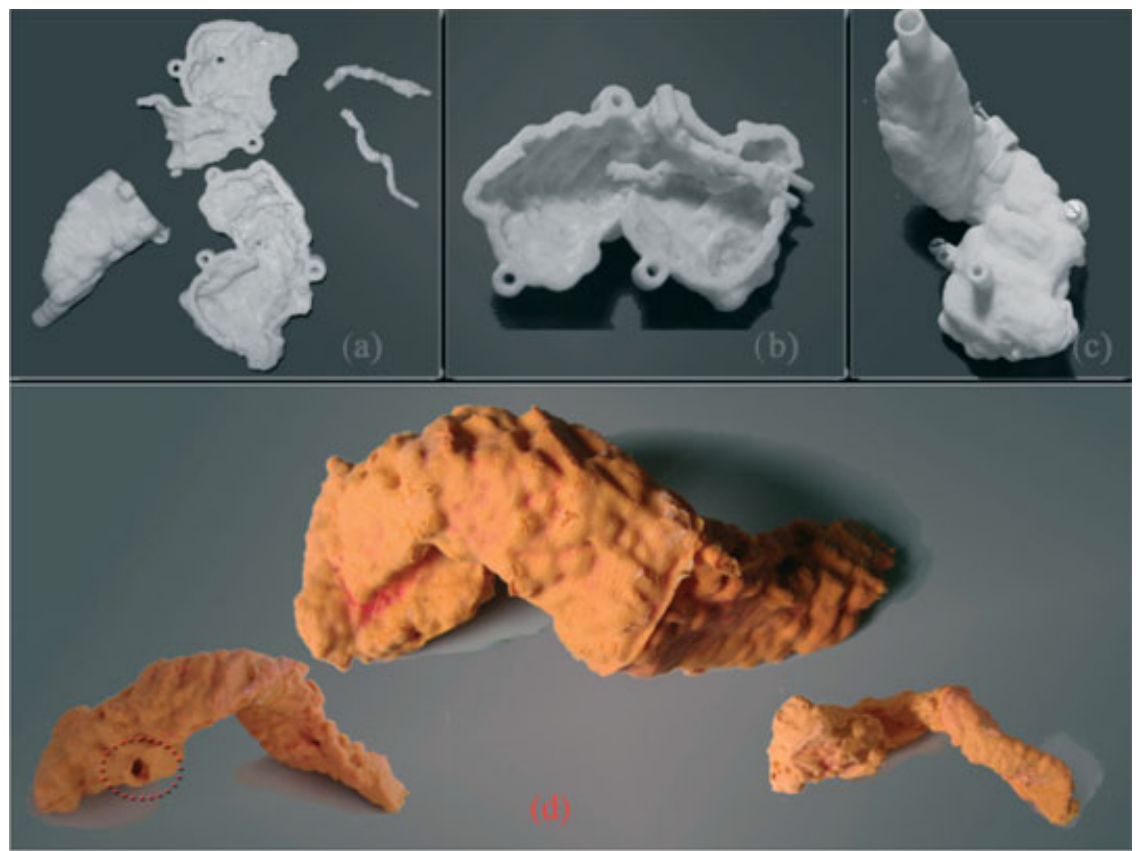

Figure 7. Pancreas fabrication process: (a) separated parts of the mould including wirsung and common bile duct abs replica; (b) wirsung and common bile duct mounted in the shell; (c) closed mould; (d) final silicone pancreas showing the common bile duct canal (red circle)
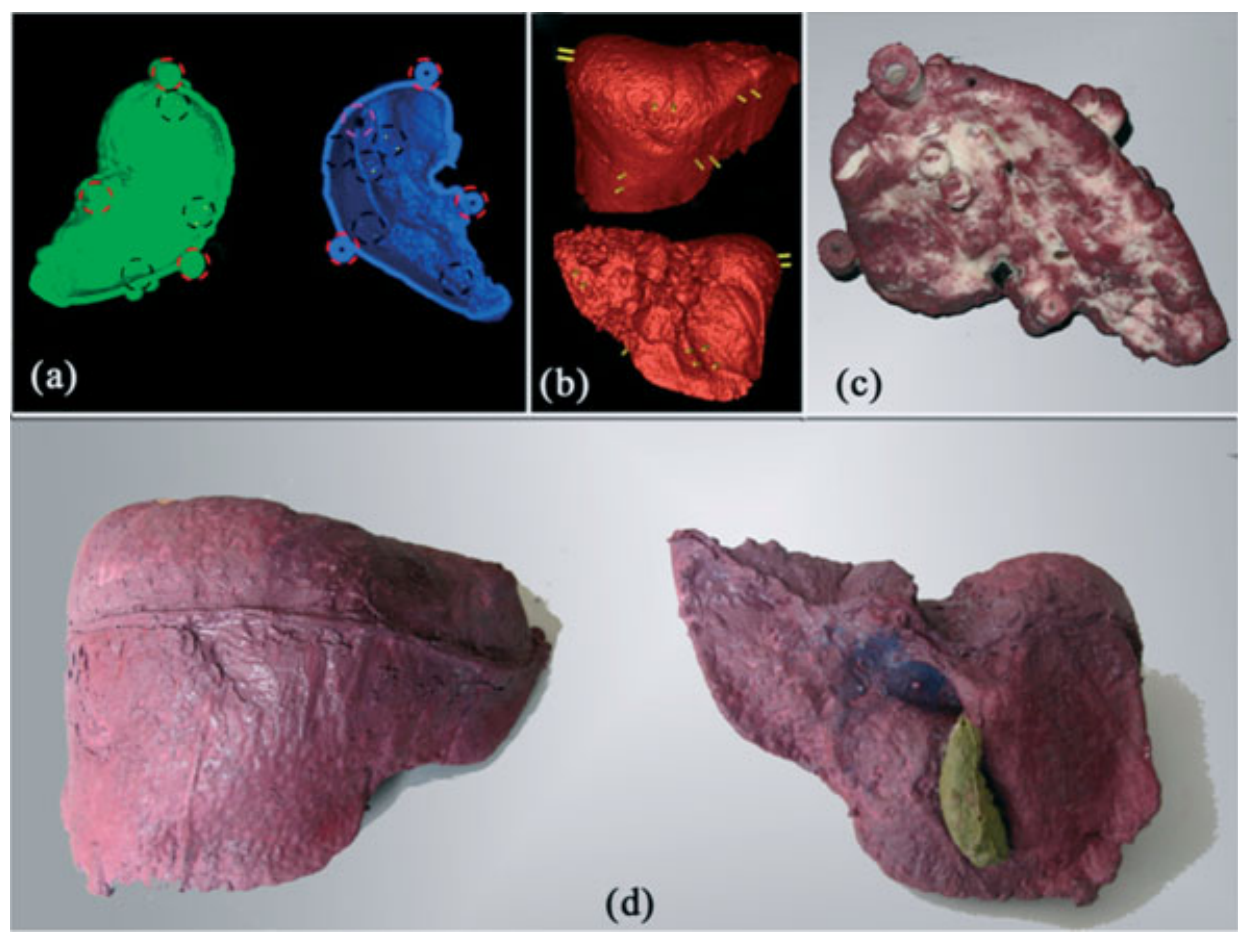

(d)

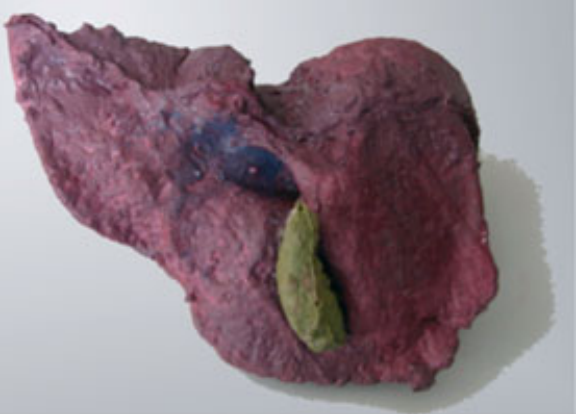

Figure 8. (a) Mould designed for liver reproduction. Red dotted circles show screw holes to guarantee proper closure of the mould. The magenta dotted circle denotes the agarose gel injection hole. (b) Selected positions for eight Aurora sensors; a screw hole predisposed in the mould parts corresponds with each cylinder. (c) Prototype mould after silicone injection. (d) Final silicone liver front (sx) and back (dx)

The following paragraphs detail the procedure for fabricating a sensorized liver model with RTV TIXO and agarose gel as filler: specifically, agarose powder from Sigma (34) (Type I-A Low EEO) mixed in water, heated until almost boiling, and then poured into the designed mould. Since the liver Young modulus varies around $20 \mathrm{kPa}(35)$ an agarose concentrations of $0.5 \%$ was used to obtain a gel with a consistent elastic modulus (31).

The mould is composed of two joinable external shells that are negative copies of the 3D liver model, Figure 8(a), 


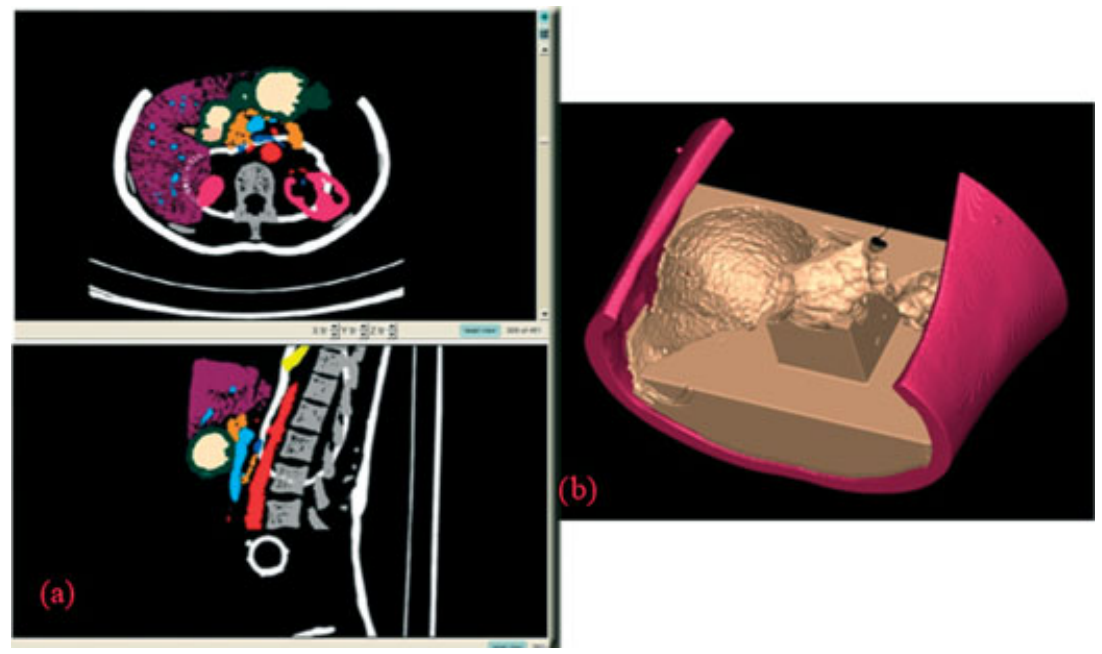

Figure 9. (a) Segmented patient organs loaded and registered on mannequin grey scale images. (b) Designed supporting structure that fits perfectly in the mannequin abdominal cavity

8(b). As for the stomach moulds, the positions for eight Aurora sensors are identified on the 3D virtual model of the liver. Figure 8(c) shows the assembled mould.

The process started with the application of a layer of RTV TIXO on the internal surface of both mould parts. Then, after silicone curing, Aurora sensors were positioned corresponding with the predisposed screws. A new layer of RTV TIXO was then applied to properly cover the sensors. When the silicone was cured, after removing the screws, the mould was closed, ensuring proper alignment of the two mould parts and using additional silicone to join the two silicone shells.

Finally, the prepared agarose gel was injected into the closed mould. The final result can be seen in Figure 8(d).

\section{Organ assembly inside the mannequin}

In order to guarantee the correct positioning of synthetic organ models inside the commercial mannequin it was decided to fabricate a supporting structure, which fits perfectly inside the mannequin, and allows the insertion of synthetic organ models, respecting their actual anatomical location in the patient. To do this, after positioning some radio opaque markers on the mannequin, a CT scan was performed. The next step was registration between patient and mannequin images: this was done using a network made in MeVisLab (36) that allows one to manually register two DICOM series. Subsequently, the registered mannequin data set was imported into our segmentation software, where the segmentation obtained from patient CT images was loaded onto the mannequin greyscale images, Figure 9(a).

This allowed segmentation of the empty space between the mannequin abdominal cavity and the organ models and thus extraction of the 3D model of a supporting structure for silicone organs that fits perfectly inside the mannequin abdomen (Figure 9(b)).

The extracted model was then refined to optimize its shape and to allow easy positioning inside the mannequin and simple insertion of the organs. Finally, the designed supporting structure was fabricated using the 3D printers.

Figure 10 shows the assembled organs inside the mannequin.

A set of abdominal walls was built to complete the simulator. These walls were added in order to simulate the pneumoperitoneum during robotic or traditional laparoscopic procedures. The covers were fabricated in thermoformable plastic material, modelled to the right shape. They are provided with soft silicone windows in strategic positions to allow the insertion of instrument access ports, Figure 10.

\section{Qualitative and quantitative tests}

The simulator was evaluated in quantitative and in qualitative terms in order to quantify its accuracy and utility, respectively.

Quantitative tests were performed to:

1. determine if the electromagnetic sensors can be damaged during silicone embedding;

2. verify the correspondence between planned and actual sensor positions, needed to perform accurate measurements of surface deformation;

3 . verify the correct positioning of synthetic organs inside the mannequin, very important to obtain correspondence between real and virtual scenarios.

The first test checks if sensors have been compromised during silicone embedding, by validating their measurement with the Aurora digitizer. A marker corresponding to each sensor centre was made on the organ surface; the digitizer tip was pushed down into the silicone at each marker. Ten acquisitions were performed for each sensor. Then, for each acquisition, the differences between values acquired by the digitizer and by the sensors were computed. 


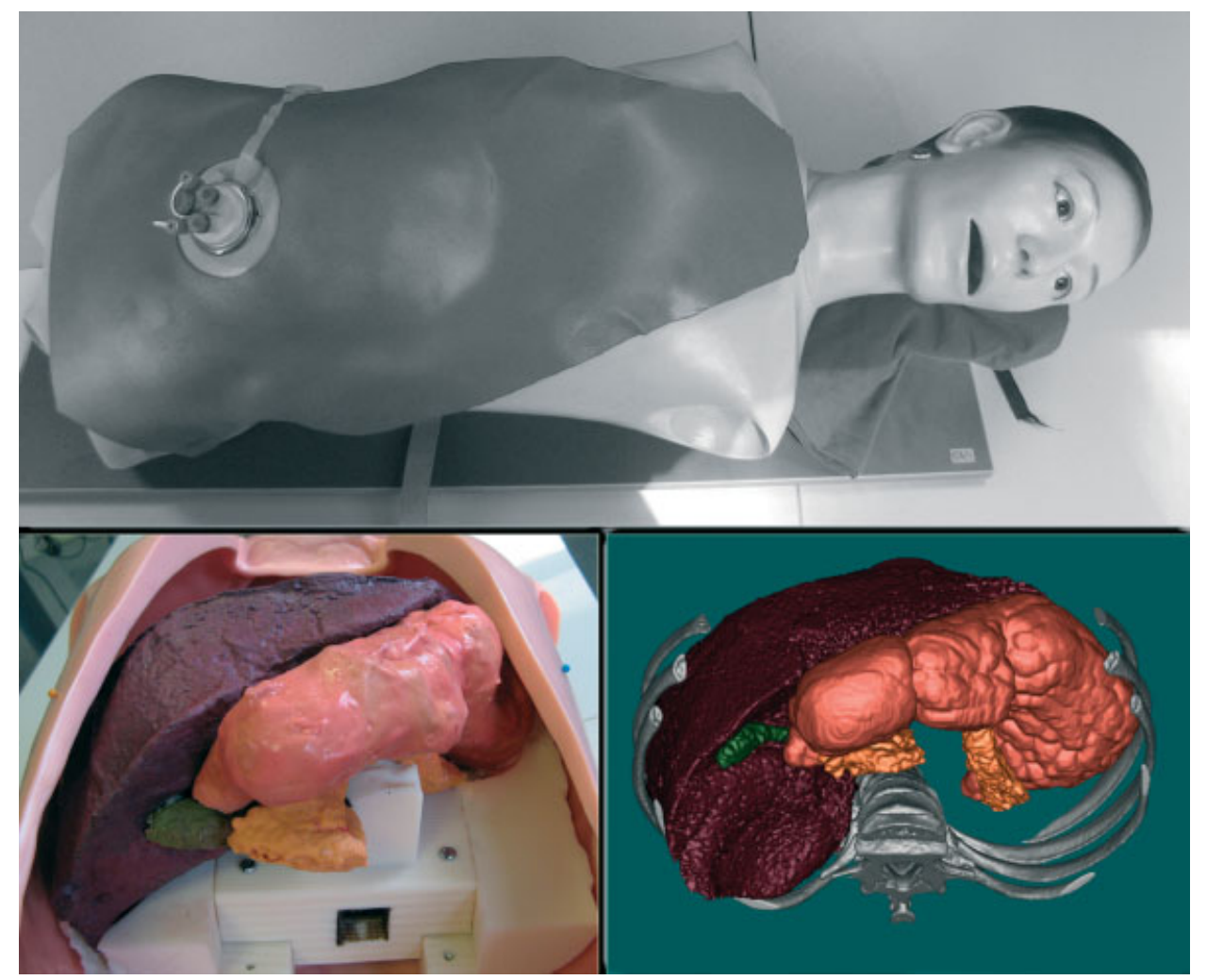

Figure 10. Assembled mannequin and the virtual models used to obtain the internal organs

The second test evaluates the precision of sensors embedding. A reference point (oesophageal sphincter centre) was selected in the CT data set and the distances between each sensor's planned position and this point were computed. The Aurora digitizer was then positioned at the silicone model sphincter and the same distances were evaluated using sensor signals. Each sensor error was evaluated by computing mean values over ten acquisitions.

Finally, a simple point based registration was applied to quantify the coherence between the virtual and the real environment. The transformation between CT and Aurora reference frames was computed using radiopaque artificial markers positioned on the mannequin. Marker positions were acquired with the Aurora digitizer, then the registration matrix was calculated through a least square error algorithm. The same registration matrix was used to register planned sensors position in the Aurora reference frame. The registered coordinates were compared with instantaneous sensor position (acquired with the localizer) to evaluate the error when positioning synthetic organs inside the mannequin.

Qualitative tests were performed to collect feedback and opinions from surgical and technical staff in terms of:

1. perceived consistency between the virtual and the synthetic environment;

2. usefulness in assessing innovative technologies;

3. potential for training purposes.

In the first test, surgical and technical staff was asked to assess the simulator accuracy by evaluating an image of the mannequin acquired with a mixed-reality platform. This test was conducted with the aim to understand how the computed accuracy errors can actually affect the perceived consistency of the simulator from the surgeons' point of view.

The mannequin virtual model was loaded in an EndoCAS surgical navigator (11) that enables mixedreality functionalities integrating a localized camera (a commercial endoscope). The least square error registration between virtual and real anatomy was computed. After that, by deploying the calibrated and localized camera, it is possible to visualize real images acquired by the camera mixed with a rendering of the virtual anatomies. Figure 11 shows a real image acquired by the endoscopic camera mixed with the rendering of the virtual anatomies obtained using the EndoCAS navigator.

The usefulness of the present simulator was first evaluated during the assessment of an innovative robotic platform for single port laparoscopy designed within the ARAKNES (Array of Robots Augmenting the KiNematics of Endoluminal Surgery) Project (37). Figure 12 shows a testing phase.

Finally, our team of surgeons was asked for their opinions on the usefulness of the simulator for surgical training. A software interface that acquires signals from the embedded sensors and emulates organ deformations on a virtual scenario (Figure 13) was implemented to show the potential offered by hybrid simulation.

Qualitative test results were collected in the form of a simple questionnaire administered to 13 clinicians. The questionnaire model is reported in Table 1. 


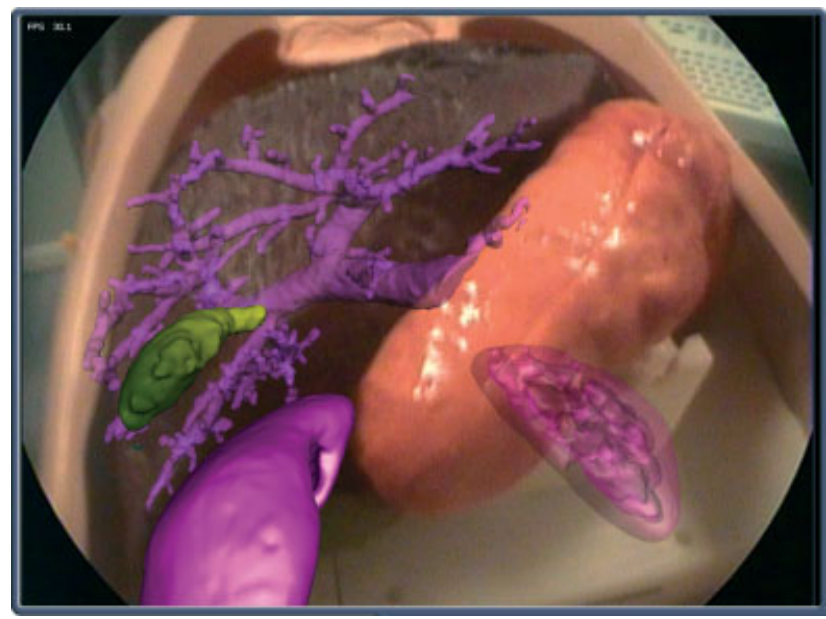

Figure 11. Screenshots of a mixed reality environment: portal system, gallbladder and kidneys are virtually mixed to the actual video streaming coming from an endoscope

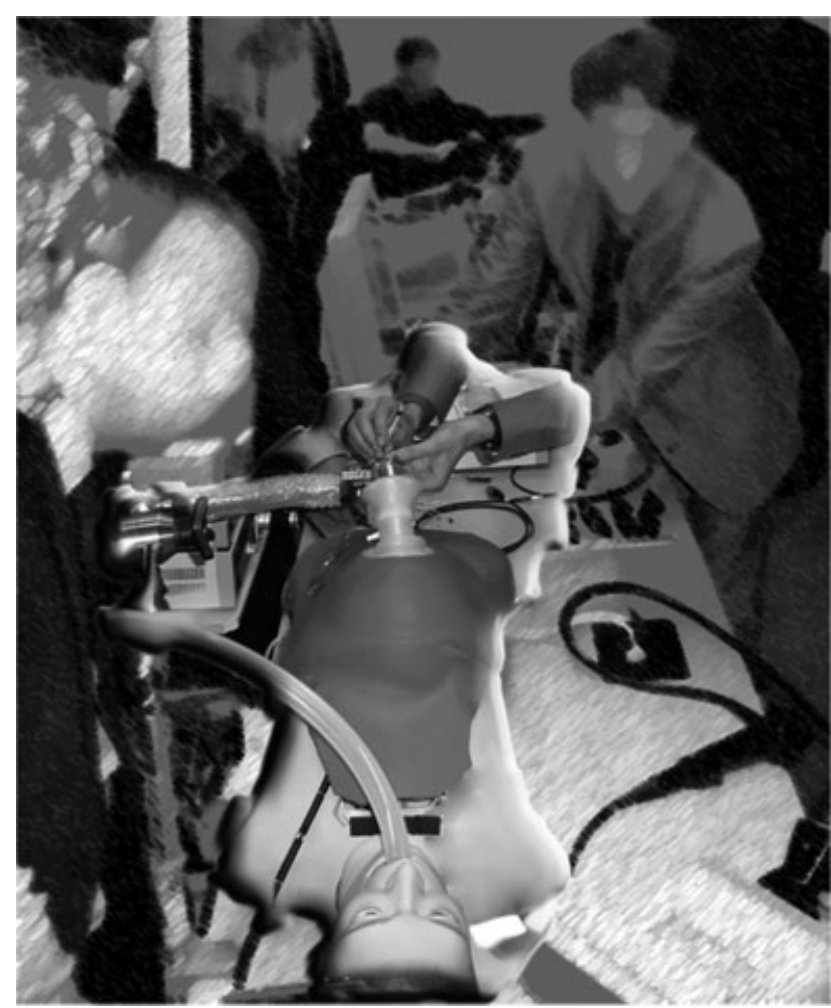

Figure 12. The simulator during the testing session of a new surgical robot

\section{Results}

The results of quantitative and qualitative tests are reported below.

The first test results show that sensors are not damaged during the silicone embedding steps because calculated errors (Table 2) are comparable with declared Aurora sensor precision (0.6 mm RMS).

The second test results are reported in Table 3 . These data validate the correspondence between planned and actual positions of sensors, needed to perform accurate measurements of surface deformation.

Regarding the last quantitative test, a final mean error of $5.2 \pm 2.1 \mathrm{~mm}$ was obtained starting from a point based registration error of $0.6199 \pm 0.1833 \mathrm{~mm}$. This error gives information about the coherence between real and virtual organ arrangements inside the mannequin. It is important to consider that this error arises from different sources: sensors positioning error (quantified in Table 3), imprecision in the acquisition of fiducial markers (fiducial localization error, which corresponds with the point based registration error), supports positioning inside the mannequin, and finally positioning of synthetic replicas on supports.

The error obtained has to be considered a worst case error since it is computed on the stomach, a big and hollow organ, which suffers more deformation than a solid organ like the liver that fits better inside the support.

Qualitative results collected with the questionnaire are reported in Table 4, while comments are summarized below for each test.

Concerning the first qualitative evaluation, the overall opinion regarding the perceived consistency between virtual and synthetic environment, despite computed errors, was very positive.

The simulator was considered very useful for assessment of the robotic platform. The comments collected underline that it is pivotal to evaluate robot workspace and movability to avoid potential collisions and damage to the surrounding anatomy. This furnishes assessed dimensional specifics for robot design.

Finally the last survey offered positive feedback on the training purpose of the physical platform.

In the first question the clinicians answered that this kind of patient-specific simulator offers the opportunity to carry out preclinical training on a copy of the patient, preparing the trainee to face the actual theatre in terms of realistic space constraints, possible instrument collisions with vital structures, and access ports placement. The last issue is a crucial time-consuming step in laparoscopic (38) surgery, where clinicians have to choose trocars placement before performing the actual intervention, and in current robotic surgery (39) where possible collisions between each robot arm have to be prevented.

Concerning evaluation of the hybrid environment, all the feedback from the surgeons was positive and they all held the opinion that this approach could be the first step in developing more complex hybrid simulation platforms, which links benefits from having a physical scenario with which to interact (mostly in terms of force feedback) with virtual elements.

These results justify the initial resources needed to fabricate the simulators. Most of the resources are invested in the primary phases, from segmentation to the rapid prototyping of moulds. Direct material costs of the moulds can be quantified around $500 € / \mathrm{kg}$ (material and soluble support), but a mould rarely reached $400 \mathrm{~g}$. The investment would be considerably higher if the rapid prototyping service was obtained from an external 


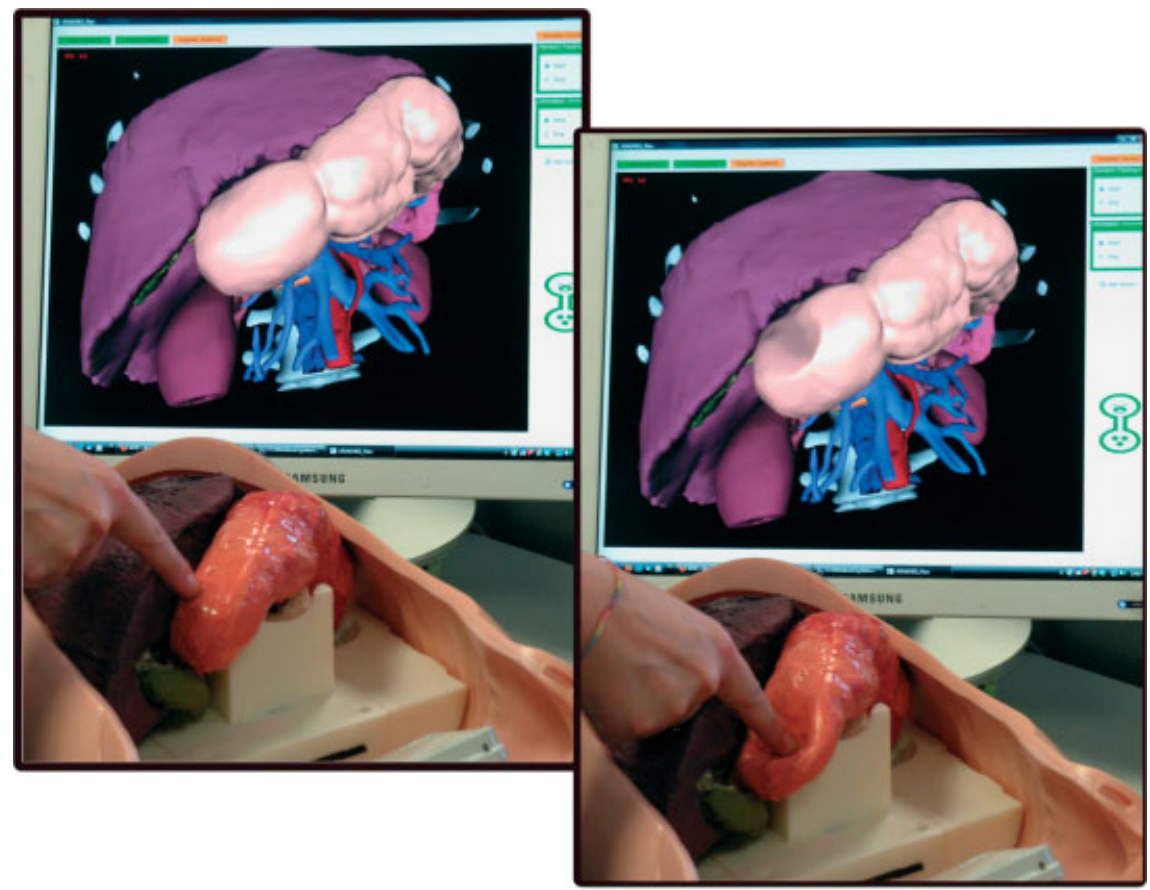

Figure 13. Example of hybrid simulation using the developed phantom

Table 1. Questionnaire administered to clinicians to qualitatively evaluate the simulator

\begin{tabular}{lll}
\hline & & \multicolumn{1}{c}{ Scoring } \\
\cline { 2 - 3 } Test 1 (mixed reality) & No (0) & Yes (1-5) \\
& $\begin{array}{l}\text { Is the simulator consistent with virtual anatomy? } \\
\text { Do you think virtual and physical anatomies are well } \\
\text { registered? How much? } \\
\text { If you perceive some misregistration issue, do you think it } \\
\text { affect simulator quality? How much? } \\
\text { Test 2 (robot testing) }\end{array}$ & $\begin{array}{l}\text { Is the simulator useful to test the robotic platform? Detail } \\
\text { which designing parameters can be assessed through the } \\
\text { phantom } \\
\text { Is the simulator useful as a training platform? Detail which } \\
\text { Tesks can be simulated. } \\
\text { Is the implemented hybrid functionality useful? Why? } \\
\text { (detail and suggest which kind of virtual add-ons could } \\
\text { enrich the simulator) }\end{array}$ \\
\hline
\end{tabular}

Table 2. Deviations along the three coordinates and mean difference between each sensor position and position acquired using the digitizer

\begin{tabular}{lcccc}
\hline $\begin{array}{l}\text { Sensor } \\
\#\end{array}$ & $\begin{array}{c}\text { Mean Err } x \\
(\mathrm{~mm})\end{array}$ & $\begin{array}{c}\text { Mean Err } \\
y(\mathrm{~mm})\end{array}$ & $\begin{array}{c}\text { Mean Err } \\
z(\mathrm{~mm})\end{array}$ & $\begin{array}{c}\text { Mean Error } \\
(\mathrm{mm})\end{array}$ \\
\hline 1 & 0.3 & 0.4 & 0.3 & 0.6 \\
2 & 0.3 & -0.1 & 0.2 & 0.4 \\
3 & 0.1 & 0.4 & 0.3 & 0.5 \\
4 & 0.2 & 0.4 & 0.3 & 0.5 \\
5 & 0.2 & -0.6 & 0.4 & 0.7 \\
6 & 0.2 & 0.2 & 0.7 & 0.7 \\
7 & -0.1 & -0.4 & -0.3 & 0.5 \\
8 & -0.1 & -0.4 & -0.6 & 0.7 \\
\hline
\end{tabular}

company. Once the moulds are built the costs are limited to the silicones ( $\max 50 € / \mathrm{kg}$ ), which is definitely affordable and economical.
Table 3. Mean distance between the position of each sensor and a reference point compared with the theoretical distance in the CT reference frame

\begin{tabular}{lccc}
\hline Sensor & $\begin{array}{c}\text { Mean Dist. Aurora } \\
(\mathrm{mm})\end{array}$ & Dist CT $(\mathrm{mm})$ & Mean Error $(\mathrm{mm})$ \\
\hline 1 & 45.1 & 45.0 & 0.1 \\
2 & 87.9 & 89.2 & 1.3 \\
3 & 125.0 & 125.3 & 0.3 \\
4 & 48.6 & 48.0 & 0.6 \\
5 & 53.1 & 52.2 & 0.9 \\
6 & 85.1 & 84.1 & 0.9 \\
7 & 66.4 & 67.7 & 1.3 \\
8 & 101.4 & 101.0 & 0.4 \\
\hline
\end{tabular}

\section{Discussion}

This paper describes a strategy to build patient-specific physical simulators with realistic shapes and colors, 
Table 4. Numerical results of the questionnaire

\begin{tabular}{|c|c|c|c|c|}
\hline & & \multirow[b]{2}{*}{ No (number of) } & \multicolumn{2}{|c|}{ Yes } \\
\hline & & & number of & mean scores \\
\hline \multirow[t]{3}{*}{ Test 1 (mixed reality) } & Is the simulator consistent with virtual anatomy? & 0 & 13 & 3.1 \\
\hline & $\begin{array}{l}\text { Do you think virtual and physical anatomies are well } \\
\text { registered? How much? }\end{array}$ & 2 & 11 & 4 \\
\hline & $\begin{array}{l}\text { If you perceive some misregistration issue, do you think it } \\
\text { affect simulator quality? How much? }\end{array}$ & 11 & 2 & 2 \\
\hline Test 2 (robot testing) & $\begin{array}{l}\text { Is the simulator useful to test the robotic platform? Detail } \\
\text { which designing parameters can be assessed through the } \\
\text { phantom }\end{array}$ & 1 & 12 & 3.5 \\
\hline \multirow[t]{2}{*}{ Test 3 (training) } & $\begin{array}{l}\text { Is the simulator useful as a training platform? Detail which } \\
\text { tasks can be simulated. }\end{array}$ & 0 & 13 & 3.9 \\
\hline & $\begin{array}{l}\text { Is the implemented hybrid functionality useful? Why? (detail } \\
\text { and suggest which kind of virtual add-ons could enrich the } \\
\text { simulator) }\end{array}$ & 0 & 13 & 4.1 \\
\hline
\end{tabular}

starting from radiological images. The strategy overcomes the limits imposed by a standard anatomy.

Our strategic approach considers that, to obtain an efficient surgical simulator, the importance of modularity and replaceability of inner structures has to be taken into account. This allows one to repeat simulation sessions in different surgical tasks and to tailor the surgical scenario to the procedure. The proposed modular method allows one to build a single organ, district or apparatus, according to the need. It is not limited to the abdominal region, the procedure can be extended to almost any other region of the body.

This procedure represents a first step towards a more complex hybrid platform that links the benefits of a physical scenario (mostly in terms of force feedback) with completely virtual elements (like hidden anatomies or bleeding vessels not reproduced in the physical phantom) and sensors information. The aim is to obtain an objective metrics of the surgical performance and to enrich the realism of the simulation. Some tests were performed to evaluate simulator accuracy and utility in quantitative and qualitative terms. Based on the results, it is possible to state that:

- consistency between the virtual and real environment is reached;

- coherence of virtual and physical organs arrangement is achieved;

- embedded sensors can be useful to estimate and emulate organ deformations.

Regarding simulator usefulness, preliminary clinical feedback confirmed that this kind of platform can be pivotal for conducting systematic pre-clinical medical assessment of innovative devices and to obtain explicit guidelines that can improve the design process. Furthermore, this simulator paves the way to a more complex hybrid platform that mixes real tactile feedback and virtual elements.

Patient-specific simulation offers young surgeons the chance to learn how to perform an entire procedure and gain improved awareness of surgical methods. While a complete evaluation of this training is currently underway, initial feedback from clinicians testing the platform are positive. This strategy to build simulators not starting from standard anatomies but describing a wide variety of anomalies and pathological scenarios is encouraged by surgeons.

\section{Acknowledgements}

This work has been financed by the European Community 'Araknes' project and by the Fondazione Cassa di Risparmio di Pisa.

\section{References}

1. Roberts KE, Bell RL, Duffy AI. Evolution of surgical skills training. World J Gastroenterol 2006; May; 12(20): 3219-3224.

2. Kneebone R, Aggarwal R. Surgical training using simulation. BMJ 2009; 338: b1001.

3. Haque S, Srinivasan S. A meta-analysis of the training effectiveness of virtual reality surgical simulators. IEEE Trans Inf Technol Biomed 2006; Jan; 10(1): 51-58.

4. Seymour NE, Rotnes JS. Challenges to the development of complex virtual reality surgical simulations. Surg Endosc 2006; Nov; 20(11): 1774-1777.

5. Botden SM, Buzink SN, Schijven MP, Jakimowicz JJ. Augmented versus virtual reality laparoscopic simulation: what is the difference? A comparison of the ProMIS augmented reality laparoscopic simulator versus LapSim virtual reality laparoscopic simulator. World J Surg 2007; Apr; 31(4): 764-772.

6. Shirai Y, Yoshida T, Shiraishi R, et al. Prospective randomized study on the use of a computer-based endoscopic simulator for training in esophagogastroduodenoscopy. J Gastroent Hepatol 2008; Jul; 23(7): 1046-1050.

7. Koch AD, Buzink SN, Heemskerk J, et al. Expert and construct validity of the Simbionix GI Mentor II endoscopy simulator for colonoscopy. Surg Endosc 2008; Jan; 22(1): 158-162.

8. Grantcharov TP, Carstensen L, Schulze S. Objective assessment of gastrointestinal endoscopy skills using a virtual reality simulator. J Soc Laparoendosc Surg 2005; Apr-Jun; 9(2): 130-133.

9. Lee JT, Qiu M, Teshome M, et al. The utility of endovascular simulation to improve technical performance and stimulate continued interest of preclinical medical students in vascular surgery. J Surg Educ 2009; Nov-Dec; 66(6): 367-373.

10. Tedesco MM, Pak JJ, Harris EJ, et al. Simulation-based endovascular skills assessment: the future of credentialing? $J$ Vasc Surg 2008; May; 47(5): 1008-1011; discussion 14.

11. Ferrari V, Megali G, Troia E, et al. A 3-D Mixed-reality system for stereoscopic visualization of medical dataset. IEEE Trans Bio-Med Eng 2009; Nov; 56(11): 2627-2633. 
12. Enquobahrie A, Gobbi D, Turek M, et al. Designing tracking software for image-guided surgery applications: IGSTK experience. Int J Comput Assist Radiol Surg 2008; Nov 1; 3(5): 395-403.

13. Yang K, Sun ZL, Kencana AP, Huynh VA, et al. Enhancement of spatial orientation and haptic perception for master-slave robotic natural orifice transluminal endoscopic surgery (NOTES). Robotics Automation Mechatron (RAM). IEEE 2010; (28-30 June 2010): 15-8.

14. Kneebone R. Simulation in surgical training: educational issues and practical implications. Med Educ 2003; Mar; 37(3): 267-277.

15. Kirk RM. Teaching the craft of operative surgery. Ann $R$ Coll Surg Eng 1996; Jan; 78(1 Suppl): 25-28.

16. SimbionixTM. www.simbionix.com.

17. Soler L, Marescaux J. Patient-specific surgical simulation. World J Surg 2008; Feb; 32(2): 208-212.

18. Suzuki S, Hattori A, Yanaga K, Suzuki N. Surgery simulation using patient-specific models for laparoscopic colectomy. Studies Health Technol Informatics 2007; 125: 464-466.

19. Cohen ZA, Henry JH, McCarthy DM, et al. Computer simulations of patellofemoral joint surgery. Patient-specific models for tuberosity transfer. Am J Sports Med 2003; Jan-Feb; 31(1): 87-98.

20. Luboz V, Ambard D, Swider P, et al. Computer assisted planning and orbital surgery: patient-related prediction of osteotomy size in proptosis reduction. Clin Biomech (Bristol, Avon) 2005; Nov; 20(9): 900-905.

21. Luboz V, Chabanas M, Swider P, Payan Y. Orbital and maxillofacial computer aided surgery: patient-specific finite element models to predict surgical outcomes. Comput Methods Biomech Biomed Eng 2005; Aug; 8(4): 259-265.

22. Pettersson J, Knutsson H, Nordqvist P, Borga M. A hip surgery simulator based on patient-specific models generated by automatic segmentation. Stud Health Technol Inform 2006; 119: 431-436.

23. Willaert WI, Aggarwal R, Nestel DF, et al. Patient-specific simulation for endovascular procedures: qualitative evaluation of the development process. Int $J$ Med Robot 2010; Jun; 6(2): $202-210$.

24. Kimura T, Morita A, Nishimura K, et al. Simulation of and training for cerebral aneurysm clipping with 3-dimensional models. Neurosurgery 2009; Oct; 65(4): 719-725.

25. Stratasys. http://www.dimensionprinting.com/.

26. NDI. http://www.ndigital.com.

27. Gossios KJ, Tsianos EV, Demou LL, et al. Use of water or air as oral contrast-media for computed tomographic study of the gastric wall - comparison of the 2 techniques. Gastrointest Radiol 1991; Fal; 16(4): 293-297.

28. Ferrari V, Cappelli C, Megali G, Pietrabissa A. An anatomy driven approach for generation of 3D models from multi-phase CT images. IJCARS Volume 3(Supplement 1):/June; 2008.

29. Yushkevich PA, Piven J, Hazlett HC, et al. User-guided 3D active contour segmentation of anatomical structures: significantly improved efficiency and reliability. Neuroimage 2006; Jul 1; 31(3): 1116-1128.

30. Prochima ${ }^{\circledR}$. www.prochima.it.

31. Bin Luo RY, Peng Ying, Michael Awad, et al, eds. Elasticity and echogenicity analysis of agarose phantoms mimicking liver tumors. Bioengineering Conference, 2006 Proceedings of the IEEE 32nd Annual Northeast 2006.

32. Wacker-Chemie. http://www.wacker.com.

33. Henry JA, O'Sullivan G, Pandit AS. Using computed tomography scans to develop an ex-vivo gastric model. World J Gastroenterol 2007; Mar 7; 13(9): 1372-1377.

34. Sigma Chemical I. http://www.sigmaaldrich.com.

35. Nava A, Mazza E, Furrer M, et al. In vivo mechanical characterization of human liver. Med Image Anal 2008; Apr; 12(2): 203-216.

36. MeVisLab. www.mevislab.de.

37. ARAKNES. www.araknes.com.

38. Ferzli GS, Fingerhut A. Trocar placement for laparoscopic abdominal procedures: a simple standardized method. J Am Coll Surg 2004; Jan; 198(1): 163-173.

39. Cannon JW, Stoll JA, Selha SD, et al. Port placement planning in robot-assisted coronary artery bypass. IEEE Trans Robotic Autom 2003; Oct; 19(5): 912-917. 